\title{
Le cours magistral en première année universitaire : des pratiques pédagogiques rénovées?
}

\author{
Amélie Duguet \\ amelie.duguet@u-bourgogne.fr
}

Article publié dans "Carrefours de l'éducation", n 45, juin 2018, pp. 93-113

La pédagogie des universitaires a, dans le contexte français, maintes fois été remise en question et les conséquences sur la scolarité des étudiants diversement interrogées. Si plusieurs écrits (Bireaud, 1990 ; Leroux, 1997) ont été produits sur le sujet au cours des années 1990, le débat est encore plus vif depuis les années 2000. Cette remise en cause intervient dans un contexte où les enseignants, du fait de la multiplicité des missions qui leur sont attribuées, se sentent tiraillés entre l'enseignement, la recherche et les tâches administratives (Musselin, 2008), et où malgré l'émergence ces dernières années des structures universitaires de pédagogie (SUP) au sein de plusieurs universités, les enseignants, si ce n'est sur la base du volontariat, sont encore peu formés à la pédagogie. Par conséquent, le modèle pédagogique qui prévaut est décrit comme étant inadapté au nouveau type de public qu'accueille l'université suite à sa démocratisation. Cette situation expliquerait d'ailleurs la fuite des étudiants pour d'autres formations de l'enseignement supérieur (Felouzis, 2003). La pédagogie universitaire soulève en effet des enjeux notamment liés au problème de l'abandon et de l'échec des étudiants, d'où la nécessité pour cette institution de réfléchir sur sa pédagogie (Paivandi, 2012). C’est d'ailleurs la raison pour laquelle différents rapports récents (Raby, 2011 ; Bertrand, 2014) recommandent une évolution des pratiques pédagogiques, par exemple en formant davantage les enseignants à la pédagogie et en valorisant la fonction pédagogique.

Ces critiques émises à l'encontre de la pédagogie universitaire concernent plus particulièrement le cours magistral. Celui-ci est vu comme faisant partie de la tradition universitaire et constituant un facteur d'échec, particulièrement au premier cycle (Leroux, 1997 ; Bruter, 2008). Sa pertinence est régulièrement remise en question (Berger, 2012). Les travaux de Bédard et Viau (2001) montrent d'ailleurs que, comparativement à d'autres situations d'enseignement comme l'approche par projet, l'étude de cas ou encore l'approche par problème, l'exposé délivré par l'enseignant, faisant référence au système traditionnel d'enseignement, n'est pas perçu comme étant très utile par les étudiants de première année. Ces derniers n'ont aucun sentiment de contrôle sur son déroulement et n'ont pas l'impression d'être capables d'apprendre durant ce type de situation pédagogique. De tels résultats s'expliquent sans doute par le fait que, depuis plusieurs décennies déjà, les cours magistraux sont décrits comme étant en grande majorité des "monologues expressifs ", des "discours qui informent sans réelle communication avec l'auditoire » (Altet, 1994, p. 37), ou bien encore un "discours oralographique " mettant face à face "un enseignant orateur et des étudiants scripteurs " (Parpette, 2002, p. 1). Quelques chercheurs ont procédé à une description plus précise des pratiques enseignantes en cours magistral. C'est notamment le cas de Clanet (2001). Menant une enquête empirique au sein de trois filières (AES ${ }^{1}$, psychologie

\footnotetext{
${ }^{1}$ Administration économique et sociale.
} 
et $\mathrm{SVT}^{2}$ ) sur trois sites universitaires (Dijon, Nantes et Toulouse), il montre que même si l'exposé oral de l'enseignant durant son intervention reste la forme d'enseignement la plus couramment utilisée, plusieurs «familles de pratiques » peuvent tout de même être mobilisées dans le cadre d'un même type de cours. À titre d'exemple, en AES la «conférence sans interaction " est employée dans 59\% des situations observées, la "conférence avec interaction » dans un peu plus d'un tiers des situations et dans une moindre mesure (5\%) une autre famille de pratiques regroupe les "cours durant lesquels ce sont les étudiants qui travaillent » (Clanet, 2001, p. 344-45). Il conclut à une réelle variété des pratiques enseignantes. Ces résultats sont congruents avec ceux de Boyer et Coridian (2002). Ces derniers, comparant des pratiques du cours magistral en première année d'histoire et de sociologie, constatent que la " conférence monologue » (Boyer, Coridian, 2002, p. 51) est la forme d'enseignement la plus courante. Ils font par ailleurs état de certaines régularités dans les pratiques enseignantes : à titre d'exemple, presque tous les enseignants sont statiques, la plupart s'appuient sur des notes et dispensent le cours de façon plutôt lente. Mais leur recherche leur permet également de mettre en avant une hétérogénéité des pratiques, observable entre les filières. Ainsi, tandis que les sociologues «tentent l'ouverture et l'interaction » (Boyer, Coridian, 2002, p. 53) avec les étudiants, les historiens, eux, préfèrent maintenir une certaine distance. Toutefois, outre des différences entre les filières, les auteurs constatent qu'il existe une variété de pratiques intra-filières. Ils avancent qu'en raison des ressources enseignantes locales, de la manière dont les départements recrutent les étudiants, et de l'absence de consensus autour de critères définissant clairement le curriculum, les enseignants sont amenés à effectuer des choix pédagogiques différents, ces choix engendrant des pratiques pédagogiques diverses.

S'ils apportent une vision plus exhaustive des pratiques des enseignants, de tels écrits restent cependant rares et datent de plus d'une décennie. Aussi cet article se propose-t-il d'apporter un éclairage récent sur les pratiques pédagogiques des enseignants en cours magistral, au travers de leur description : quelle est la nature de ces pratiques ? Sur quelles méthodes pédagogiques reposent-elles ? Existe-t-il une variété dans ces pratiques ? De telles questions demeurent dans un contexte où les travaux dénonçant un modèle pédagogique universitaire inadapté à l'hétérogénéité du public étudiant se multiplient, et où le gouvernement actuel affiche une volonté politique de rénover la pédagogie universitaire. Nous focalisons notre attention sur les cours ayant lieu en première année universitaire, étant celle la plus touchée par le problème de l'échec des étudiants. Toutefois, avant d'apporter des éléments de réponse à ces questionnements, il convient de définir ce que recouvrent les concepts de « méthode » et de « pratique » pédagogique.

\section{Des méthodes aux pratiques pédagogiques : positionnement théorique}

Les méthodes en pédagogie sont nombreuses, comme en témoigne la longue liste dressée par Raynal et Rieunier (2005): méthodes impositives, traditionnelles, attrayantes, intuitives, actives, démonstratives, méthode interrogative, globale, nouvelle, méthodes centrées sur le contenu, modernes, audiovisuelles, en sont des exemples. En réalité, le passage en revue de la littérature scientifique sur le sujet témoigne d'un manque de consensus autour de ce que recouvre réellement ce terme. Nous retiendrons néanmoins la définition de Raynal et Rieunier (2005), selon qui elles consistent en une « organisation codifiée de techniques et de moyens ayant pour but de faciliter l'action éducative » (Raynal, Rieunier, 2005, p. 227). En d'autres

\footnotetext{
${ }^{2}$ Sciences de la vie et de la terre
} 
termes, elles «rassemblent les acteurs et définissent les caractéristiques matérielles, cognitives et sociales d'une pratique pédagogique " (Morandi, La Borderie, 2006, p. 126). Différents auteurs ont établi des typologies des méthodes. C'est par exemple le cas de Prégent (1990) qui réunit 34 méthodes d'enseignement en trois catégories : les méthodes fondées sur l'exposé, celles fondées sur la discussion et le travail d'équipe, et celles fondées sur l'apprentissage individuel. Cette classification laisse entrevoir l'existence de trois grands types de méthodes: le premier repose sur un schéma transmissif de l'enseignant vers l'étudiant, le deuxième met l'accent sur le travail de groupe et le dernier sur l'apprenant. En réalité, la plupart des chercheurs, à l'image d'Arénilla, Gossot, Rolland et Roussel (2000), ou bien encore de Raynal et Rieunier (2005), s’accordent à replacer les méthodes sur un continuum allant d'une conception «magistro-centrée ${ }^{3}$ " de l'enseignement, lesquelles méthodes sont souvent qualifiées de "traditionnelles", à une conception centrée sur l’apprenant, faisant ainsi référence aux méthodes « actives ».

Par ailleurs, les méthodes constituent «des guides », « des organisateurs du travail pédagogique » (Morandi, 2001, p. 12). Certes, la méthode ne détermine pas entièrement les pratiques d'un enseignant puisque « la pratique échappe toujours un tant soit peu à la théorie » (Houssaye, 2009, p. 13). Elle oriente néanmoins le choix des pratiques pédagogiques (Bru, 2006). La pratique pédagogique, concept faisant souvent l'objet d'un amalgame avec celui de méthode et de modèle pédagogique, consiste, elle, à "mettre en place un certain nombre de conditions cognitives, matérielles, relationnelles, temporelles auxquelles les élèves sont confrontés » (Bru, 2006, p. 117). En référence à ces travaux, nous définissons les pratiques pédagogiques comme représentant toutes les actions mises en œuvre par l'enseignant, de manière plus ou moins consciente, en vue de faire acquérir des connaissances aux étudiants et pouvant se rapporter à plusieurs dimensions. Ainsi, nous identifions la façon qu'a l'enseignant de transmettre le cours, sa clarté dans sa manière de le faire et son organisation du cours, parmi les dimensions se référant aux conditions cognitives mises en place par l'enseignant et mentionnées par Bru (2006). La façon de transmettre le cours fait référence à la manière dont l'enseignant s'appuie sur des supports en vue de communiquer le cours (lecture de feuilles, dictée du cours ...), au rythme du cours ainsi qu'à l'emploi de pratiques telles que l'humour, le récit d'anecdotes ou encore l'utilisation d'exemples. La clarté est relative tout autant aux pratiques destinées à faciliter l'appréhension du cours et la prise de notes par l'étudiant (dictée du cours, reformulation, accent mis sur les points principaux du cours, explicitation des nouveaux termes techniques utilisés...), qu'aux pratiques mises en œuvre pour appuyer les étudiants dans leur travail personnel (explicitation des modalités d'examen, consignes de travail). L'organisation désigne la manière dont l'enseignant planifie les séances et articule les savoirs pour les faire acquérir aux étudiants (présentation du but du cours, récapitulatif de ce qui a été vu la fois précédente, gestion du volume horaire, alternance entre cours magistral et travail individuel...). Le matériel utilisé par l'enseignant reprend les conditions pareillement nommées par Bru. Il fait ainsi référence aux supports de cours employés par l'enseignant. Enfin, les interactions avec les étudiants et l'attitude de l'enseignant sont des dimensions que nous prenons également en considération car elles permettent de se pencher notamment sur l'aspect relationnel évoqué par Bru (2006). Les interactions peuvent être considérées sous plusieurs angles : celui de leur fréquence et de leur motif. L'attitude renvoie quant à elle à la manière qu'a l'enseignant de s'exprimer, à ses déplacements dans l'amphithéâtre ou encore à sa conduite corporelle (gestes, mimes).

\footnotetext{
${ }^{3}$ Ce terme emprunté à Frenay (2006) est repris par Demougeot-Lebel et Perret (2010, p.57) et désigne un modèle d'enseignement transmissif dans lequel l'enseignant privilégie les contenus.
} 
Au vu de la littérature mentionnée, nous formulons trois hypothèses :

- La première est que les pratiques des enseignants en cours magistral s'appuient en grande majorité sur les méthodes «traditionnelles » telles qu'elles sont définies par Bru (2006), c'est-à-dire comme reposant « sur un processus de transmission par lequel le savoir est directement donné aux élèves », sensés "l'enregistrer et l'accumuler » (Bru, 2006, p. 33). En d'autres termes, nous supposons qu'elles reposent sur un schéma selon lequel l'enseignant est le principal détenteur des connaissances qu'il «dispense » aux étudiants. Nous nous attendons donc notamment à ce que les enseignants se montrent statiques, dictent le cours, interagisssent peu ou pas avec les étudiants, utilisent peu de matériel pour enseigner.

- La seconde hypothèse est qu'il existe une variété dans les pratiques pédagogiques mobilisées par les enseignants en cours magistral. Autrement dit, bien que s’appuyant sur les mêmes méthodes, tous les enseignants n'emploieraient pas les mêmes pratiques, que cela soit entre les filières ou bien au sein d'une seule et même filière. A titre d'exemple, nous nous attendons à ce que certains mobilisent le diaporama pour enseigner, tandis que d'autres utiliseront plutôt un tableau avec une craie.

- La troisième hypothèse, énoncée en référence aux travaux de Bru, vise à considérer que les étudiants n'ont pas une opinion massivement négative des pratiques de leurs enseignants.

\section{Présentation de la recherche empirique}

Cette recherche a pour originalité de ne pas avoir abordé les pratiques des enseignants telles qu'elles sont déclarées par les enseignants, mais telles qu'elles peuvent être observées in situ. Pour répondre aux objectifs de cette recherche, nous avons donc procédé à l'observation des pratiques de 49 enseignants exerçant en cours magistral dans une université française. Certes, il aurait été préférable d'investiguer au sein de toutes les filières. Cependant, des contraintes matérielles évidentes nous ont conduite à restreindre notre terrain d'enquête à cinq filières : Droit, $\mathrm{AES}^{4}$, Sociologie, Psychologie et $\mathrm{LLCE}^{5}$ Anglais. Nous ne nous étendrons pas sur les caractéristiques de ces différentes filières car ce n'est pas l'objet du travail présenté ici. Notons néanmoins que celles-ci tendent toutes à relever du domaine des sciences humaines, à tendance appliquée. Mais le choix de ces filières se justifie par le fait qu'elles présentent plusieurs intérêts: elles accueillent un grand nombre d'étudiants ${ }^{6}$, présentant une hétérogénéité à la fois en termes d'origine sociale et de passé scolaire et proposent un nombre important de cours magistraux lors des deux semestres.

\footnotetext{
${ }^{4}$ Administration économique et sociale

${ }^{5}$ Langues, littératures et civilisations étrangères

${ }^{6}$ La population de référence inscrite dans ces filières en première année en 2012-2013 est de 143 individus en LLCE Anglais, 380 en AES, 854 en Droit, 149 en sociologie et 473 en psychologie. En raison de la non assiduité de certains d'entre eux, nous n'avons en revanche aucune donnée concernant le nombre d'étudiants effectivement présents en cours à chaque séance.
} 
Comme l’indique Bruter (2008), le terme « cours magistral » ${ }^{7}$, souvent désigné comme un mode d'enseignement dans lequel un professeur expose son savoir devant un auditoire, peut être ambivalent: le nombre d'étudiants présents en cours, le lieu du cours, la fonction supposée de transmission des connaissances peuvent en être autant d'éléments de définition. De ce fait, il est ici considéré au sens institutionnel du terme, tel qu'il est administrativement qualifié dans les maquettes de formation.

Pour procéder aux observations, nous avons construit une grille d'observation (voir annexe 1) constituée des dimensions des pratiques mentionnées dans notre définition du terme. Chaque dimension était composée d'items destinés à traduire des pratiques. Certes, différents items sont sujets à la subjectivité de l'observateur. Il s'agit là cependant de l'un des écueils assumé de la méthode de l'observation, qui permet néanmoins de repérer certaines tendances. Les observations ont eu lieu en octobre et mars de l'année universitaire 2012-2013. Afin de consolider la mesure, les pratiques de chaque enseignant ont été observées à deux reprises lors de séances de cours entières, c'est-à-dire soit pendant une heure, soit pendant deux heures, selon le format horaire du cours.

Pour avoir une vue plus synthétique de ces pratiques, nous avons ensuite calculé pour chaque enseignant un score moyen sur 100 pour chaque dimension de la grille d'observation ${ }^{8}$. Plus le score est proche de 100, plus il signifie que l'enseignant a adopté des pratiques relatives à la dimension nommée. La description des pratiques repose en partie sur l'analyse de ces scores.

Compte tenu du faible nombre d'individus que comprend l'échantillon d'enseignants et afin de préserver l'anonymat de ces derniers, nous raisonnons sur l'ensemble des filières. Précisons toutefois que les enseignants sont majoritairement des hommes (27/49) et que les maîtres de conférences (30 individus) et les professeurs (12 individus) sont les plus représentés, les autres étant professeurs agrégés (3), attaché temporaire à l'enseignement et à la recherche (1), doctorant allocataire (1) ou vacataires (2).

\section{Des pratiques issues des méthodes traditionnelles}

Les box plot du graphique ci-dessous illustrent la dispersion des scores relatifs à chacune des dimensions des pratiques. Plus le corps de la boîte est petit, plus les valeurs de la distribution sont homogènes. Au contraire, plus il est grand, plus les valeurs sont dispersées. Les extrêmes représentent la valeur minimale et maximale de la distribution. Le corps de la boîte fournit les valeurs correspondant au premier et au troisième quartile, ainsi qu’à la médiane.

\section{Graphique 1 : Dispersion des scores des enseignants de l'échantillon en fonction des dimensions des pratiques pédagogiques.}

\footnotetext{
${ }^{7}$ Les dimensions épistémologique et historique du cours magistral ne constituant pas l'objet central de notre propos, nous ne reviendrons pas ici sur ces dernières. Nous invitons pour cela le lecteur à se référer à l'ouvrage de J. Houssaye (2014) : La pédagogie traditionnelle. Une histoire de la pédagogie. Paris : Éditions Fabert.

${ }^{8}$ La construction de ces scores est davantage détaillée dans l’annexe 1.
} 


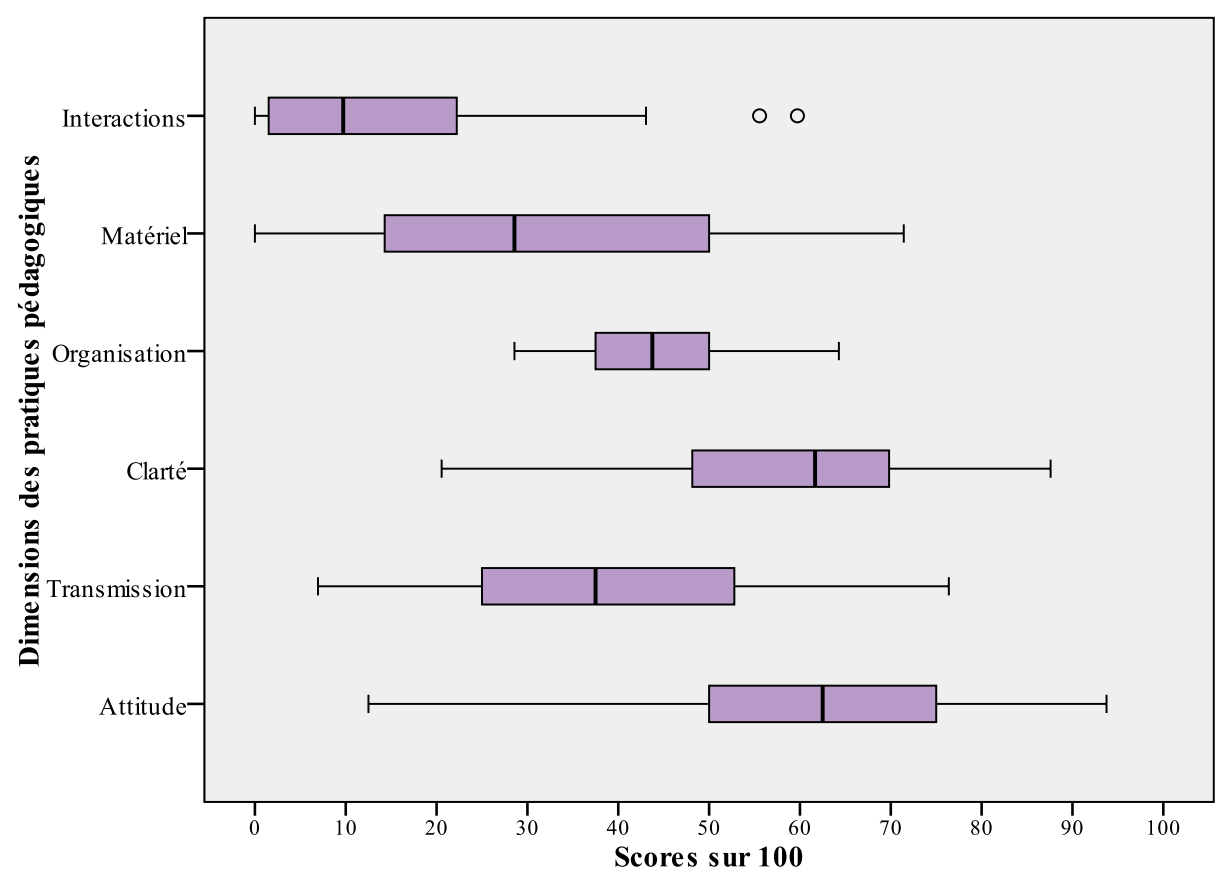

À la lecture de ce graphique, on constate que la dimension interactive est la moins privilégiée par les enseignants : les scores vont de 0 à 59,72, la médiane se situant à 9,72. Les échanges verbaux entre enseignants et étudiants sont peu nombreux : plus de $85 \%$ interagissent assez rarement, voire jamais avec les étudiants. Comme avait déjà pu le mettre en exergue Altet (1994), les questions posées par les enseignants sont généralement d'ordre rhétorique : à peine plus d'un sur deux laisse le temps de réfléchir aux étudiants lorsqu'il pose une question. Presque aucun n'a requis l'avis des étudiants sur le cours ou la méthode employée. Cette rareté des interactions constitue l'une des caractéristiques des méthodes pédagogiques traditionnelles qui consistent, d'après Arénilla, Gossot, Rolland et Roussel (2000), en la transmission du savoir de façon expositive. En outre, près de 6 enseignants sur 10 ont dicté le cours aux étudiants et plus des trois quarts n’ont pas alterné durant les séances entre cours magistral et travail individuel ou en groupe de la part des étudiants. Certes, la seule observation des cours magistraux ne suffit pas à rendre compte de l'ensemble des pratiques pédagogiques employées par les enseignants, puisqu'il est probable qu'une telle pratique soit davantage mobilisée lors des travaux dirigés. Mais il n'en demeure pas moins que peu d'enseignants ont rendu les étudiants réellement acteurs de leurs apprentissages lors des cours magistraux. Or, selon Bireaud (1990), la méthode traditionnelle génère le plus souvent « une situation d'enseignement principalement collectif où le travail personnel est laissé sous la complète responsabilité de l'étudiant ».

Le graphique présenté ci-dessus permet de mettre en avant un autre résultat intéressant : la médiane du score lié à la dimension du matériel, se situant à 28,57, est elle aussi relativement faible. Cela signifie que les enseignants utilisent dans l'ensemble peu de matériel dans le cadre de leurs pratiques pédagogiques. La distribution du plan du cours, d'une bibliographie ou bien d'autres polycopiés représente une pratique minoritaire (un tiers voire moins des enseignants de l'échantillon). Par ailleurs, alors que Bireaud évoquait déjà en 1990 toute l'importance d'avoir recours aux nouvelles technologies pour contribuer à la rénovation des pratiques pédagogiques, et à l'heure où le Ministère de l'Enseignement Supérieur et de la Recherche entrevoit les TICE comme pouvant contribuer à la rénovation des pratiques des enseignants, ce matériel reste concernant les enseignants de l'échantillon plutôt classique. L’usage du tableau (27 enseignants) est plus répandu que celui de la présentation PowerPoint 
(13 enseignants). Ces résultats conduisent à s’interroger sur la place des nouvelles technologies au sein des cours magistraux : leur usage paraît peu développé. On peut alors se demander si cette situation est plutôt liée à une absence de moyens matériels fournis par l'institution, à une méconnaissance de ces technologies numériques par les enseignants ou encore à une réticence de leur part, notamment liée à une absence de formation en la matière, concernant l'emploi de ce type d'outils. Toutefois, nous nous sommes penchée sur les pratiques mobilisées durant les cours uniquement. Il est probable que certaines pratiques relatives aux nouvelles technologies, telle la mise en ligne des cours sur une plateforme pédagogique, interviennent en dehors des heures de cours. Cette faible utilisation des outils numériques contraste d'ailleurs avec la recherche de Loizon et Mayen (2015), qui eux, indiquent à l'issu d'une recherche empirique que tous les enseignants font usage d'une présentation power-point en cours magistral. Ils expliquent néanmoins leurs résultats par un effet lié à la culture propre des écoles d’ingénieurs au sein desquelles ils ont investigué.

Par ailleurs, Arénilla et al. (2000), ont mentionné comme figurant parmi les méthodes traditionnelles "l'enseignement frontal ", à travers lequel l'enseignant, sur son estrade, inculque les savoirs à une classe disposée en rangs alignés. Or, cette vision d'un statisme de l'enseignant est aussi apparente parmi les individus de l'échantillon. En effet, la moyenne de la dimension liée à l'attitude $(61,7$, écart-type à 17,4$)$ tend à témoigner d'un score élevé pour cette dimension, cela en raison du fait que la grande majorité des enseignants parlent distinctement, sachent se faire entendre au fond de la salle et regardent leur public. Néanmoins, seuls 4 enseignants se sont déplacés dans l'amphithéâtre lors des séances de cours, faisant écho aux écrits d'Arénilla et al. (2000) sur les méthodes traditionnelles.

De même, les résultats liés à la clarté des enseignants indiquent certes que plus de 9 enseignants sur 10 répètent et reformulent régulièrement leurs propos. Ils sont en revanche à peine plus de la moitié à mettre l'accent sur les points principaux pour aider les étudiants à les identifier et moins d'un quart à avoir fourni des consignes aux étudiants, ces consignes n'étant en outre pas toujours précises. De tels résultats entrent en résonnance avec la définition de Raynal et Rieunier (2005), selon qui les méthodes traditionnelles consistent en « l'acceptation du principe selon lequel le rôle du maître consiste à dispenser le savoir, l'élève devant s'organiser au mieux pour l'apprendre » (Raynal, Rieunier, 2005, p. 277), laissant ainsi entendre qu'il incombe à l'étudiant de trouver seul des solutions pour s'organiser dans le travail personnel qu'il a à réaliser.

De tels résultats tendent à aller dans le sens de la validation de notre première hypothèse et sont en ce sens comparables à ceux d'Altet (1994), Clanet (2001) ou encore Boyer et Coridian (2002). Ils laissent à penser que les pratiques des enseignants en cours magistral n'ont que peu évolué durant cette dernière décennie. Néanmoins, plusieurs explications peuvent venir justifier l'usage de telles pratiques. La première a trait à la formation à la pédagogie des enseignants qui est, à l'échelle nationale, peu développée. De ce fait, on peut imaginer que nombre d'entre eux tendent à reproduire, par effet de mimétisme, les pratiques de leurs pairs et respectent une tradition implicite à laquelle il est difficile de déroger en vue de rester dans la «norme ». D'autres raisons sont, elles, de nature plus contextuelle. Il est probable que certains, ayant pourtant une vision puedo-centrée de l'enseignement, n'aient d'autres possibilité que de mettre en place des pratiques à visée transmissive, en raison des conditions matérielles qui leur sont octroyées. À titre d'exemple, les cours magistraux se déroulent dans la majorité des cas dans des amphithéâtres, dans lesquels sièges et autres tables sont en rangs alignés cloués au sol, entravant ainsi le déplacement du matériel pour agencer la salle différemment. Il en est de même du micro qui est le plus souvent vissé au bureau, facilitant 
peu les déplacements de l'enseignant dans l'amphithéâtre. Il serait de ce fait intéressant d'examiner s'il existe une concordance entre les objectifs visés par les enseignants et les pratiques qu'ils mettent en œuvre pour les atteindre. De plus, on peut supposer que la taille des groupes influe sur la mise en œuvre de ce type de pratiques, Crespo et Houle (1995) mentionnant le fait qu'on a souvent tendance à recourir aux méthodes traditionnelles pour faire face aux grands groupes. On peut en outre évoquer un manque de temps pour approfondir leur réflexion sur leurs pratiques, puisque les enseignants exerçant à l'université sont partagés entre la recherche, les tâches administratives et l'enseignement, cette dernière mission faisant d'ailleurs l'objet d'une faible valorisation de la part de l'institution.

Finalement, le maintien et même la reproduction de ce type de pratiques se situe au cœur de plusieurs enjeux pour l'institution universitaire. L'un d'entre eux concerne la dimension financière. Un changement s’appuyant sur d'autres méthodes pédagogiques nécessiterait vraisemblablement que les enseignants soient davantage formés à la pédagogie, aient à disposition d'autres types de matériel pour enseigner, ou bien encore que la taille des groupes soit réduite, représentant des investissements conséquents pour les universités. Un autre type d'enjeu est de nature plus institutionnelle : il s'agirait de déroger à une tradition ancestrale, autrefois sans doute valorisée, selon laquelle durant le cours magistral l'enseignant est le " magister » au sens de celui qui détient les savoirs et qui dirige. Toutefois, des questions se posent également concernant la variété des pratiques des enseignants.

\section{La variété des pratiques pédagogiques en cours magistral}

L'observation des pratiques des enseignants a permis de constater que celles-ci n'étaient en rien immuables. D'abord, une même pratique n'est pas systématiquement employée d'une séance de cours à l'autre. A titre d'exemple, un focus sur les pratiques relatives à l'organisation du cours montre qu'au cours de la première séance durant laquelle leurs pratiques ont été observées, 11 enseignants ont présenté le but du cours en début de séance. Ils sont 18 dans ce cas lors de la seconde séance d'observation. Des enseignants font de ce fait preuve de variabilité dans l'usage de cette pratique selon les séances de cours. Entrent sans doute en jeu ici certaines variables de nature affective, comme l'humeur et la motivation de l'enseignant. La nature du contenu des cours, variant d'une semaine à l'autre, constitue elle aussi certainement un facteur explicatif.

De même, certaines pratiques sont employées différemment en fonction des séances. Par exemple, durant la première séance au cours de laquelle leurs pratiques ont été observées, 7 enseignants ont rarement répété leurs propos, 16 l'ont fait souvent et 20 continuellement. Ces chiffres varient en ce qui concerne la seconde séance d'observation : 10 ont employé cette pratique rarement, 20 souvent et 13 continuellement. Des enseignants, bien que faisant usage de cette pratique lors de chaque cours, n’y ont pas recours toujours à la même fréquence.

Mais il existe également une hétérogénéité des pratiques entre les enseignants. Le calcul des scores sur 100 pour chaque dimension des pratiques a abouti à obtenir 26 scores différents concernant la dimension des interactions avec les étudiants, 8 pour l'utilisation du matériel, 11 pour l'organisation, 37 pour la clarté, 30 pour la façon de transmettre le cours et enfin 21 pour l'attitude de l'enseignant. Autrement dit, si l'on prend l'exemple de la dimension liée aux interactions, les enseignants sont au moins 26 à ne pas avoir employé les mêmes pratiques que leurs collègues, puisque leur score diffère des autres. Cela ne signifie cependant pas que les 23 enseignants restants ont mis en œuvre des pratiques identiques, puisqu'il est possible que 
deux enseignants aient obtenu un même score, sans pour autant employer les mêmes pratiques. De même, les box-plot présentés dans la partie ci-dessus montrent que les scores des enseignants concernant les différentes dimensions des pratiques sont relativement étendus. À titre d'exemple, celui lié à l'attitude varie de 12,50 à 93,75, tandis que celui relatif à l'utilisation du matériel s'étend de 0 à 71,43 . Ces résultats témoignent d'une grande variabilité des valeurs.

La dispersion des scores peut également être appréhendée à travers l'observation des écartstypes comme il en est le cas dans le tableau suivant :

Tableau 1 : Moyenne et écart-type des scores pour chacune des six dimensions des pratiques pédagogiques

\begin{tabular}{|c|c|c|c|c|c|c|}
\cline { 2 - 7 } \multicolumn{1}{c|}{} & $\begin{array}{c}\text { Interactions } \\
\text { avec les } \\
\text { étudiants }\end{array}$ & $\begin{array}{c}\text { Utilisation } \\
\text { du } \\
\text { matériel }\end{array}$ & Organisation & Clarté & $\begin{array}{c}\text { Façon de } \\
\text { transmettre } \\
\text { le cours }\end{array}$ & Attitude \\
\hline $\begin{array}{c}\text { Score } \\
\text { moyen }\end{array}$ & 16,16 & 35,71 & 44,33 & 58,68 & 39,14 & 61,72 \\
\hline $\begin{array}{c}\text { Ecart- } \\
\text { type }\end{array}$ & 16,36 & 19,78 & 9,48 & 15,27 & 17,18 & 17,40 \\
\hline
\end{tabular}

Les dimensions liées à l'attitude, à la façon de transmettre le cours et à l'utilisation du matériel présentent une plus grande variabilité de scores. C'est concernant cette dernière dimension que les scores sont les plus dispersés autour de la moyenne : 68\% des enseignants ont un score compris entre 15,93 et 55,49. La dispersion liée aux interactions avec les étudiants et à la clarté est elles aussi non négligeable. En revanche, un écart-type de 9,48 pour la dimension relative à l'organisation du cours indique une moindre dispersion.

Conformément à notre seconde hypothèse, nos résultats témoignent d'une variété des pratiques enseignantes, rejoignant en cela les conclusions de Clanet (2001). Cette diversité est telle que nous n'avons pu établir de typologie des enseignants en fonction des pratiques mobilisées. En effet, dans le but de créer des catégories d'enseignants, nous avons procédé à une analyse en composantes principales. Le test de Kaiser-Meyer-Olkin, avec une mesure s'élevant à 0,636, indique une validité faible. L'analyse n'a en outre permis de dégager que deux composantes difficilement interprétables. Se pose alors la question des motifs pouvant contribuer à expliquer cette variété. L'une des premières raisons tient sans doute à la formation à la pédagogie des enseignants. Du fait de la diversité de leur statut (Professeur, Maître de Conférences, ATER, PRAG, doctorant allocataire...), tous n'ont vraisemblablement pas été formés de la même manière et n’ont de surcroît pas le même vécu ni la même expérience en matière de pédagogie. Il semble alors cohérent de constater que tous n'emploient par les mêmes pratiques. D'autres facteurs explicatifs se rattachent davantage au contexte d'enseignement. L'enquête a été conduite au sein de cinq filières. Il est probable que les ressources pédagogiques mises à disposition des enseignants, en termes de matériel notamment, ne soient pas les mêmes au sein de chacune d'entre elles. On peut de même penser que la filière et la discipline enseignée interviennent en partie dans l'explication de cette hétérogénéité des pratiques. À titre d'exemple, peut-on réellement enseigner à l'appui des mêmes pratiques un cours de traduction en anglais qu'un cours d'histoire du droit ? Finalement, bien que nous avancions ici différentes hypothèses pour expliquer ces résultats, prolonger ce travail empirique par des entretiens auprès des enseignants permettrait 
certainement d'apporter des éléments de réponse à ces questionnements et de concevoir de nouvelles pistes explicatives concernant les choix (si tant est que l'on puisse parler d'un choix) de pratiques des enseignants.

\section{Conclusion}

Déjà en 1994 Altet évoquait le fait que «le cours magistral est devenu inadapté au public étudiant actuel et pose aujourd'hui des problèmes pédagogiques graves qu'il devient urgent de traiter faute d'augmenter l'échec à l'université » (Altet, 1994, p. 39). Des travaux témoignent d'une préoccupation grandissante autour de la question de la rénovation de ce type de cours. C'est ainsi que des recherches ${ }^{9}$ ont été produites sur des dispositifs destinés à dynamiser les cours magistraux et à accroître les interactions entre les enseignants et les étudiants. A la lecture de ces écrits, nous nous sommes interrogée, plus d'une décennie après les travaux de Clanet (2001) et Boyer et Coridian (2001), sur les pratiques effectives des enseignants en cours magistral. Nous avons pour cela procédé à l'observation in situ des pratiques de 49 enseignants issus de cinq filières différentes. Nos résultats témoignent d'un maintien de pratiques issues des méthodes traditionnelles, c'est-à-dire centrées sur l'enseignant, ou autrement dit reposant sur un schéma transmissif. Néanmoins, nous avons pu démontrer dans un même temps que ces pratiques présentaient une certaine hétérogénéité intra et inter enseignants, dont il serait intéressant de dégager plus finement la part liée aux différences de pratiques inter et intra-filières.

Comme il en est le cas pour toute recherche empirique, le travail exposé ici se heurte à différentes limites, méthodologiques notamment. Ainsi, il serait sans doute probant de repenser la grille d'observation des pratiques, notamment du point de vue du choix des intitulés des dimensions. Celui-ci a été effectué de façon arbitraire, s'agissant là de l'un des écueils assumés de la recherche. Il nous est apparu a posteriori que certains termes, tels que «l'attitude ", pouvaient faire référence à des concepts précis en psychologie sociale. Une redéfinition des dimensions permettrait donc de compléter le travail présenté ici. De plus, des analyses de cohérence interne, qui n’ont pu être réalisées pour ce premier travail exploratoire, permettraient de vérifier que les différents items d'une même dimension mesurent la même chose et ont des poids équivalents. Par ailleurs, une discussion sur la validité des scores présentés pourrait être engagée, la constitution de ces derniers demandant à être affinée. Notre approche quantitative a du reste tendance à absorber en partie la finesse des informations recueillies. En outre, comme évoqué précédemment, il serait intéressant de davantage tenir compte, pour expliquer les pratiques des enseignants, des spécificités des disciplines, mais aussi et surtout des savoirs enseignés au moment des observations.

Néanmoins, les résultats mis au jour présentent l'intérêt d'alimenter la réflexion sur la rénovation du cours magistral. Comme l'évoque Raby (2011), il est probable que cette rénovation passe nécessairement par une revalorisation de la fonction pédagogique et par là même une institutionnalisation au plan national de la formation, initiale et continue, des enseignants à la pédagogie. Toutefois, on peut supposer qu'elle est également dépendante d'une rénovation du contexte, matériel notamment, qui conditionne le déroulement des cours magistraux. Se pose d'ailleurs ici la question de l'apport des outils numériques. Leur

\footnotetext{
${ }_{9}^{9}$ À l'image de ceux de Leclercq, Gibbs et Jenkins (1998) et Leclercq, Denis, Jans, Poumay et Gilles
} (1998) 
mobilisation pour enseigner est préconisée par le gouvernement qui entrevoit le numérique comme un moyen de faire évoluer les pratiques des enseignants universitaires. Loizon et Mayen (2015) indiquent que «les diaporamas numériques, l'usage d'Internet in situ avec les étudiants et les fichiers numériques sont autant d'instruments qui s'ajoutent ou se substituent à la prestation orale de l'enseignant ». On peut toutefois s'interroger sur la nature de la fonction pédagogique qu'attribuent les enseignants à ces outils. Une enquête pourrait en ce sens être menée, afin d'examiner si les outils numériques conduisent réellement à un changement de paradigme induisant la mobilisation de pratiques davantage actives.

Amélie Duguet

Institut de recherche sur l'éducation (IREDU - ÉA7318)

Université de Bourgogne Franche-Comté (UBFC)

\section{Bibliographie}

Altet M. (1994). Le cours magistral universitaire : un discours scientifico-pédagogique sans articulation enseignement-apprentissage. Recherche et Formation, n¹5, p. 35-44.

Arénilla L., Gossot B., Rolland M. C., Roussel M. P. (2000). Dictionnaire de pédagogie. Paris : Bordas.

Bédard D., Viau R. (2001). Le profil d'apprentissage des étudiantes et des étudiants de l'université de Sherbrooke. Résultats de l'enquête menée au trimestre d'automne 2000. Manuscrit non publié, Université de Sherbrooke, Vice-rectorat à l'enseignement. Sherbrooke

Berger V. (2012). Assises de l'enseignement supérieur et de la recherche. Rapport au président de la République, 17 décembre 2012.

Bertrand, C. (2014). Soutenir la transformation pédagogique dans l'enseignement supérieur. Paris : Ministère de l’Enseignement Supérieur et de la Recherche.

Bireaud A. (1990). Les méthodes pédagogiques dans l'enseignement supérieur. Paris: Editions d'organisation.

Boyer R., Coridian C. (2002). Transmission des savoirs disciplinaires dans l'enseignement universitaire, une comparaison histoire-sociologie. Sociétés contemporaines, n48, p. 41-61.

Bru M. (2006). Les méthodes en pédagogie. Paris : Presses Universitaires de France.

Bruter A. (2008). Le cours magistral comme objet d'histoire. Histoire de l'éducation, $\mathrm{n}^{\circ} 120$, p. 5-32.

Clanet J. (2001). Étude des organisateurs des pratiques enseignantes à l'université. Revue des Sciences de l'Éducation, vol. 27, n²2, p. 327-352.

Crespo M., Houle R. (1995). La persévérance aux études dans les programmes de premier cycle à l'Université de Montréal. Manuscrit inédit. Université de Montréal : vice-décanat aux études supérieures et à la recherche.

Demougeot-Lebel J., Perret C. (2010). Identifier les conceptions de l'enseignement et de l'apprentissage pour accompagner le développement professionnel des enseignants débutants à l'université. Savoirs, n²3, p. 51-72. 
Felouzis G. (2003). Les mutations actuelles de l'Université. Paris : Presses Universitaires de France.

Houssaye J. (2009). Le triangle pédagogique ou comment comprendre la situation pédagogique. In J. Houssaye (dir.). La pédagogie, une encyclopédie pour aujourd'hui. Paris : ESF, p. 13-25.

Leclercq D., Denis B., Jans V., Poumay M., Gilles J.L. (1998). L'amphithéâtre électronique. Une application : le LQRT-SAFE. In : D. Leclercq (dir.). Pour une pédagogie universitaire de qualité. Sprimont : Mardaga éd, p. 161-186.

Leclercq D., Gibbs G., Jenkins A. Le défi des grands groupes. (1998). In D. Leclercq (dir.). Pour une pédagogie universitaire de qualité. Sprimont : Mardaga éd, p.137-159.

Leroux J.Y. (1997). Les premiers cycles universitaires français dans le contexte de l'enseignement supérieur de masse. Gestion de l'enseignement supérieur, vol. 9, nº1, p. 115125.

Loizon A., Mayen P. (2015). Le cours magistral en amphithéâtre : une situation d’enseignement perturbée par les instruments. Distances et Médiations des Savoirs, n³.

Morandi F. (2001). Modèles et méthodes en pédagogie (2 éd.). Paris: Nathan.

Morandi F., La Borderie R. (2006). Dictionnaire de pédagogie. Paris: Nathan.

Musselin C. (2008). Les universitaires. Paris : La Découverte.

Paivandi S. (2012). Pour réussir la transition entre secondaire et supérieur. Cahiers pédagogiques, Hors série numérique, n²5, p. 22-25.

Parpette C. (2002). Le cours magistral, un discours oralographique : effet de la prise de notes des étudiants sur la construction du discours de l'enseignant. In dans R. Gauthier \& A. Meggori (eds.) Actes du colloque Langages et significations : L'oralité dans l'écrit et réciproquement, Albi, p. 261-266.

Prégent R. (1990). La préparation d'un cours. Montréal : Éditions de l’École Polytechnique.

Raby, G. (2011). Pour une licence attractive doublement qualifiante, permettant une poursuite d'études en master et une insertion professionnelle, une licence assurant un parcours de réussite aux étudiants. Contribution écrite au colloque annuel de la Conférence des Présidents d'université, Toulouse, 11-12-13 mai. En ligne http://inpact.inptoulouse.fr/CPU2011/contributions \%20ecrites.html, consulté le 3 juin 2011.

Raynal F., Rieunier A. (2005). Pédagogie, dictionnaire des concepts clés : apprentissages, formation, psychologie cognitive. Paris : ESF. 


\section{Annexes}

\section{Annexe 1 : La construction des scores de pratiques pédagogiques}

Pour construire ces scores, nous avons attribué pour chaque item de 0 à 3 points selon l'observation effectuée. À titre d'exemple, pour l'item «de façon générale, l'enseignant interagit avec les étudiants ", nous avons attribué 0 point lorsque l’observateur avait coché la case non, 1 point lorsqu'il avait entouré les fréquences 1 ou 2, 2 points pour les fréquences 3 et 4 , et 3 points pour les fréquences 5 et 6 . De même, pour les items composés de deux possibilités de réponse, tel que "il répond aux questions en cas d'incompréhension ", nous avons attribué 3 points lorsque l'enseignant employait ce type de pratique durant son cours et 0 point lorsque la pratique n’était pas mobilisée. Aucune variable n’a été pondérée dans la constitution de ces scores : nous avons fait le choix d'accorder la même importance à toutes les variables mobilisées dans la constitution de ces scores. Ceux-ci ont ensuite été standardisés sur 100.

\section{Annexe 2 : Grille d'observation des pratiques}


GRILLE D'OBSERVATION

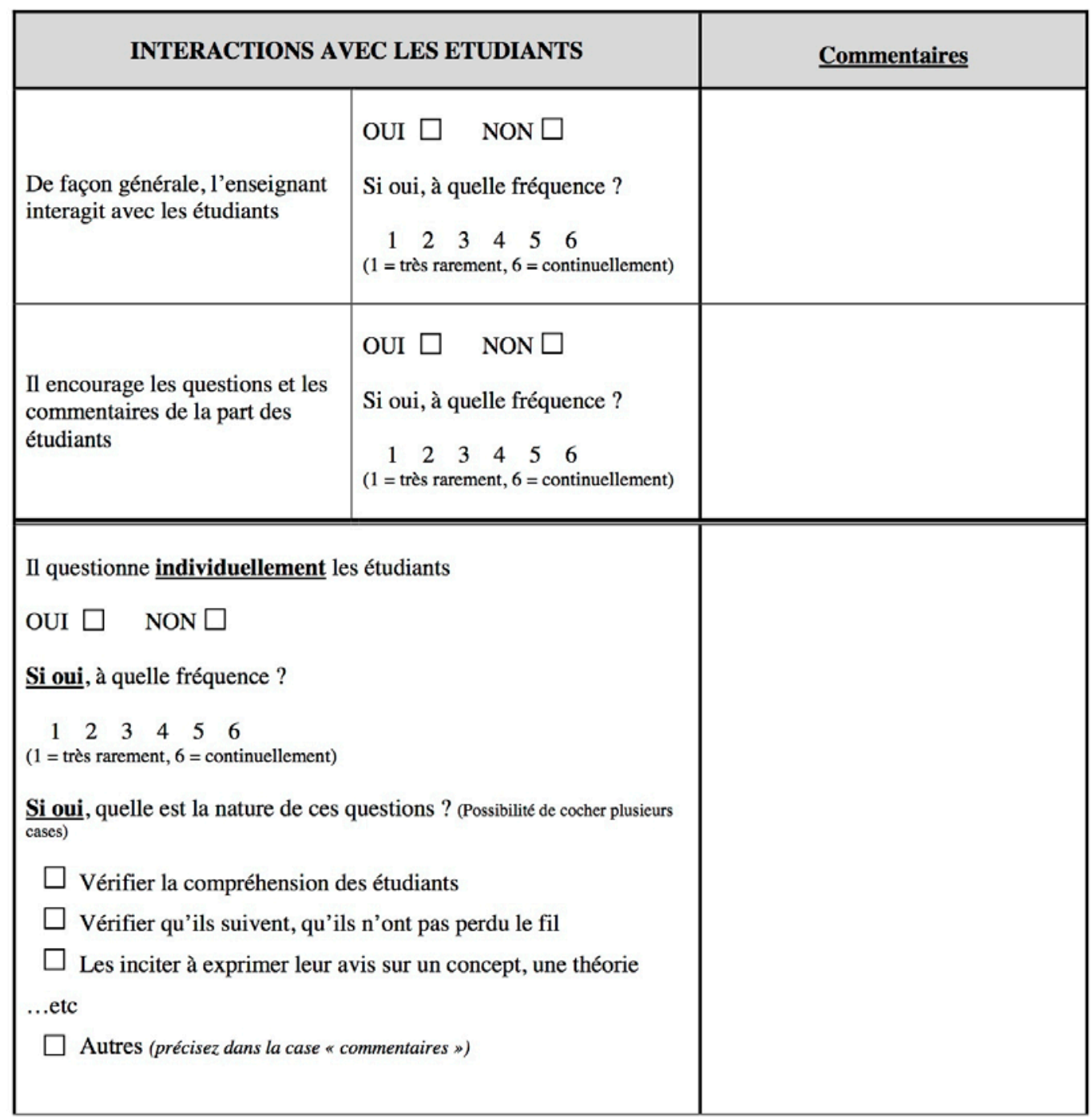


Il pose des questions au groupe entier

\section{OUI $\square \quad$ NON $\square$}

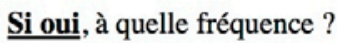

$\begin{array}{llllll}1 & 2 & 3 & 4 & 5 & 6\end{array}$

( 1 = très rarement, 6 = continuellement $)$

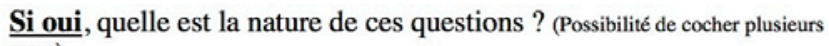
cases)

$\square$ Vérifier la compréhension des étudiants

$\square$ Vérifier qu'ils suivent, qu'ils n'ont pas perdu le fil

$\square$ Les inciter à exprimer leur avis sur un concept, une théorie ...etc

$\square$ Autres (précisez dans la case « commentaires »)

Il laisse le temps de la réflexion après avoir posé une question

Il demande aux étudiants si tout est bien compris avis sur le cours
OUI $\square \quad$ NON $\square$

OUI $\square \quad$ NON $\square$

$\square$ OUI (concernant la méthode employée)

$\square$ OUI (concernant les thèmes abordés et le contenu du cours)

$\square$ NON

\begin{tabular}{|c|c|c|}
\hline \multicolumn{2}{|c|}{ UTILISATION DU MATERIEL } & Commentaires \\
\hline L'enseignant utilise un power-point & OUI $\square \quad$ NON $\square$ & \\
\hline \multicolumn{2}{|c|}{ Si utilisation d'un power-point : } & \\
\hline Nombre de diapos : & 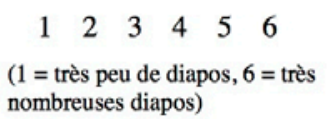 & \\
\hline Vitesse de défilement des diapos : & $\begin{array}{l}\begin{array}{cccccc}1 & 2 & 3 & 4 & 5 & 6 \\
(1=\text { très lentement, } 6=\text { très } \\
\text { rapidement })\end{array}\end{array}$ & \\
\hline
\end{tabular}




\begin{tabular}{|c|c|c|}
\hline $\begin{array}{l}\text { Le power-point contient l'intégralité } \\
\text { de ce que dit l'enseignant }\end{array}$ & OUI $\square \quad$ NON $\square$ & \\
\hline $\begin{array}{l}\text { L'enseignant a intégré des schémas } \\
\text { dans son power-point }\end{array}$ & OUI $\square \quad$ NON $\square$ & \\
\hline$\underline{\text { Si oui, ces schémas sont-ils clairs? }}$ & OUI $\square$ & \\
\hline L'enseignant utilise le tableau & OUI $\square \quad$ NON $\square$ & \\
\hline \multicolumn{3}{|c|}{ Si utilisation du tableau : } \\
\hline $\begin{array}{l}\text { L'enseignant note le plan du cours } \\
\text { au tableau }\end{array}$ & OUI $\square \quad$ NON $\square$ & \\
\hline $\begin{array}{l}\text { L'enseignant note des références, } \\
\text { noms des auteurs, mots compliqués, } \\
\text { phrases clés }\end{array}$ & $\begin{array}{l}\text { OUI } \square \\
\text { Si oui, à quelle fréquence ? } \\
\begin{array}{ccccccc}1 & 2 & 3 & 4 & 5 & 6\end{array} \\
\begin{array}{l}(1=\text { très rarement, } 6= \\
\text { continuellement })\end{array}\end{array}$ & \\
\hline $\begin{array}{l}\text { L'enseignant fait des schémas } \\
\text { Si oui, ces schémas sont-ils clairs? }\end{array}$ & $\begin{array}{l}\text { OUI } \square \quad \text { NON } \square \\
\text { OUI } \square \quad \text { NON } \square \\
\end{array}$ & \\
\hline $\begin{array}{l}\text { L'enseignant fournit (ou a fourni) un } \\
\text { plan du cours }\end{array}$ & $\begin{array}{l}\square \text { OUI (sous forme papier) } \\
\square \text { oUI (sous forme orale) } \\
\square \text { NON }\end{array}$ & \\
\hline $\begin{array}{l}\text { L'enseignant fournit (ou a fourni) } \\
\text { une bibliographie }\end{array}$ & $\begin{array}{l}\square \text { OUI (sous forme papier) } \\
\square \text { OUI (sous forme orale) } \\
\square \text { NON }\end{array}$ & \\
\hline $\begin{array}{l}\text { L'enseignant distribue des } \\
\text { polycopiés autres que le plan du } \\
\text { cours et la bibliographie (articles } \\
\text { scientifiques, cours...) }\end{array}$ & OUI $\square \quad$ NON $\square$ & \\
\hline $\begin{array}{l}\text { Si utilisation de polycopiés, ceux-ci } \\
\text { sont lus en cours }\end{array}$ & OUI $\square \quad$ NON $\square$ & \\
\hline $\begin{array}{l}\text { Si utilisation de polycopiés, ceux-ci } \\
\text { servent de base au cours }\end{array}$ & OUI $\square \quad$ NON $\square$ & \\
\hline $\begin{array}{l}\text { L'enseignant utilise un micro } \\
\text { (Précisez dans la case commentaire s'il n'y a } \\
\text { pas de micro) }\end{array}$ & OUI $\square \quad$ NON $\square$ & \\
\hline
\end{tabular}




\begin{tabular}{|c|c|c|c|}
\hline \multicolumn{3}{|c|}{ ORGANISATION } & Commentaires \\
\hline \multicolumn{3}{|c|}{$\begin{array}{l}\text { Au début de la séance, l'enseignant présente le but du cours: } \\
\text { OUI } \square \quad \text { NON } \square\end{array}$} & \\
\hline $\begin{array}{l}\text { En début de séance, il fait un } \\
\text { récapitulatif de ce qui a été vu la fois } \\
\text { précédente }\end{array}$ & OUI $\square$ & NON $\square$ & \\
\hline $\begin{array}{l}\text { Au début du cours, il demande aux } \\
\text { étudiants ce qu'ils ont retenu de la } \\
\text { fois précédente }\end{array}$ & OUI $\square$ & NON $\square$ & \\
\hline $\begin{array}{l}\text { Il respecte le volume horaire dont il } \\
\text { dispose } \\
\text { (Pas de notion développée e à la va vite » juste } \\
\text { avant la fin du cours car n'a plus suffisamment de } \\
\text { temps, volume horaire entierement rempli..) }\end{array}$ & OUI $\square$ & NON $\square$ & \\
\hline $\begin{array}{l}\text { En fin de séance, il fait un résumé } \\
\text { des notions qui ont été abordées }\end{array}$ & OUI $\square$ & NON $\square$ & \\
\hline $\begin{array}{l}\text { Il accorde une pause aux étudiants } \\
\text { entre } 2 \text { heures de cours }\end{array}$ & OUI $\square$ & NON $\square$ & \\
\hline $\begin{array}{l}\text { Il alterne entre cours magistral et } \\
\text { travail individuel ou en groupe de la } \\
\text { part des étudiants }\end{array}$ & OUI $\square$ & NON $\square$ & \\
\hline $\begin{array}{l}\text { Il organise son cours de façon claire } \\
\text { et structurée }\end{array}$ & OUI $\square$ & NON $\square$ & \\
\hline
\end{tabular}

\begin{tabular}{|c|c|c|}
\hline \multicolumn{2}{|c|}{ CLARTE } & Commentaires \\
\hline $\begin{array}{l}\text { Il répète plusieurs fois les } \\
\text { choses } \\
\text { (Pour s'assurer que les étudiants ont } \\
\text { compris et ont eu le temps de prendre } \\
\text { des notes) }\end{array}$ & $\begin{array}{l}\text { OUI } \square \\
\text { Si oui, à quelle fréquence ? } \\
\begin{array}{llllll}1 & 2 & 3 & 4 & 5 & 6\end{array} \\
\left(\begin{array}{l}1=\text { très rarement, } 6=\text { continuellement })\end{array}\right.\end{array}$ & \\
\hline $\begin{array}{l}\text { Il reformule plusieurs fois de } \\
\text { façons différentes ses propos }\end{array}$ & $\begin{array}{l}\text { OUI } \square \\
\text { Si oui, à quelle fréquence ? } \\
\begin{array}{llllll}1 & 2 & 3 & 4 & 5 & 6\end{array} \\
\left(\begin{array}{l}1=\text { très rarement, } 6=\text { continuellement })\end{array}\right.\end{array}$ & \\
\hline
\end{tabular}




\begin{tabular}{|l|l|l|}
\hline $\begin{array}{l}\text { Il met l'accent sur les points } \\
\text { centraux pour aider les } \\
\text { étudiants à les identifier }\end{array}$ & OUI $\square$ NON $\square$ & \\
\hline $\begin{array}{l}\text { Il explique tout nouveau terme } \\
\text { technique utilisé }\end{array}$ & OUI $\square \quad$ NON $\square$ & \\
\hline $\begin{array}{l}\text { Il répond aux questions en cas } \\
\text { d'incompréhension }\end{array}$ & OUI $\square \quad$ NON $\square$ & \\
\hline $\begin{array}{l}\text { Il utilise un langage clair, } \\
\text { facile à comprendre }\end{array}$ & OUI $\square \quad$ NON $\square$ & \\
\hline $\begin{array}{l}\text { Il explique (ou a expliqué) les } \\
\text { modalités d'examen aux } \\
\text { étudiants }\end{array}$ & OUI $\square \quad$ NON $\square$ & \\
\hline $\begin{array}{l}\text { Il fournit des consignes aux } \\
\text { étudiants afin que ceux-ci } \\
\text { sachent comment travailler } \\
\text { leurs cours une fois chez eux } \\
\text { Si oui, ces consignes sont elles } \\
\text { précises? }\end{array}$ & OUI $\square \quad$ NON $\square$ & \\
\hline
\end{tabular}

\begin{tabular}{|c|c|c|}
\hline \multicolumn{2}{|c|}{ FACON DE TRANSMETTRE LE COURS } & \multirow[t]{2}{*}{ Commentaires } \\
\hline L'enseignant lit ses feuilles & OUI $\square \quad$ NON $\square$ & \\
\hline Il lit son power-point & OUI $\square \quad$ NON $\square$ & \\
\hline Il dicte le cours & $\begin{array}{l}\text { OUI } \square \\
\text { Si oui, à quelle fréquence ? } \\
\begin{array}{cccccc}1 & 2 & 3 & 4 & 5 & 6 \\
(1=\text { très rarement, } 6=\text { continuellement })\end{array}\end{array}$ & \\
\hline $\begin{array}{l}\text { Il fait des pauses quand il } \\
\text { parle pour laisser aux } \\
\text { étudiants le temps de prendre } \\
\text { des notes }\end{array}$ & $\begin{array}{l}\text { OUI } \square \\
\text { Si oui, à quelle fréquence ? } \\
\begin{array}{cccccc}1 & 2 & 3 & 4 & 5 & 6\end{array} \\
(1=\text { très rarement, } 6=\text { continuellement })\end{array}$ & \\
\hline Il raconte des anecdotes & OUI $\square \quad$ NON $\square$ & \\
\hline Il utilise l'humour & OUI $\square \quad$ NON $\square$ & \\
\hline Il utilise des exemples & OUI $\square \quad$ NON $\square$ & \\
\hline
\end{tabular}




\begin{tabular}{|l|l|l|}
\hline & OUI $\square \quad$ NON $\square$ & \\
Il recommande des lectures & $\begin{array}{c}\text { Si oui, à quelle fréquence ? } \\
1 \quad 2 \quad 3 \quad 4 \quad 5 \quad 6 \\
\begin{array}{l}1=\text { très rarement, } 6=\text { continuellement })\end{array}\end{array}$ \\
\hline Rythme & $\begin{array}{c}1 \\
(1=\text { très lent, } 6=\text { très rapide })\end{array}$ & \\
\hline Il raconte une «histoire » & OUI $\square \quad$ NON $\square$ & \\
\hline Il essaie de garder l'attention & OUI $\square \quad$ NON $\square$ & \\
\hline
\end{tabular}

\begin{tabular}{|l|l|l|}
\hline \multicolumn{2}{|c|}{ ATTITUDE } & Commentaires \\
\hline $\begin{array}{l}\text { L'enseignant parle d'une voix } \\
\text { monotone }\end{array}$ & OUI $\square \quad$ NON $\square$ & \\
\hline Il parle distinctement & OUI $\square \quad$ NON $\square$ & \\
\hline $\begin{array}{l}\text { Il parle fort, sait se faire entendre } \\
\text { au fond de la salle }\end{array}$ & OUI $\square \quad$ NON $\square$ & \\
\hline $\begin{array}{l}\text { Il utilise le langage corporel (gestes } \\
\text { et mimes) }\end{array}$ & OUI $\square \quad$ NON $\square$ & \\
\hline $\begin{array}{l}\text { Il se montre enthousiaste, } \\
\text { dynamique }\end{array}$ & OUI $\square \quad$ NON $\square$ & \\
\hline $\begin{array}{l}\text { Il se déplace dans tout } \\
\text { l'amphithêtre }\end{array}$ & OUI $\square \quad$ NON $\square$ & \\
\hline Il regarde son public & OUI $\square \quad$ NON $\square$ & \\
\hline $\begin{array}{l}\text { Il maintient l'ordre et la discipline } \\
\text { si besoin }\end{array}$ & OUI $\square \quad$ NON $\square$ & \\
\hline
\end{tabular}

